\title{
The structure of non-nilpotent CTI-groups
}

\author{
Hamid Mousavi, Tahereh Rastgoo and Viktor Zenkov \\ Communicated by Evgeny I. Khukhro
}

\begin{abstract}
A subgroup $H$ of a group $G$ is called a TI-subgroup if $H \cap H^{g} \in\{1, H\}$, for all $g \in G$, and a group is called a CTI-group if all of its cyclic subgroups are TI-subgroups. In this paper, we determine the structure of non-nilpotent CTI-groups. Also we will show that if $G$ is a nilpotent CTI-group, then $G$ is either a Hamiltonian group or a non-abelian p-group.
\end{abstract}

\section{Introduction and preliminaries}

Throughout the following, $G$ always denotes a finite group.

Let $H$ be a subgroup of $G$. If for every $g \in G$ we have $H \cap H^{g} \in\{1, H\}$, then $H$ is called a TI-subgroup. Now if every subgroup of $G$ is a TI-subgroup, then $G$ is called a TI-group, and $G$ is an ATI-group if all of its abelian subgroups are TI-subgroups. In [13], G. Walls classified the TI-groups. S. Li and X. Guo in [6] classified the ATI-groups of prime power order; also these authors with P. Flavell in [4] determined the structure of ATI-groups.

A subgroup $H$ of $G$ is called a QTI-subgroup if for every $1 \neq x \in H$, we have

$$
\digamma_{G}(x) \leq \mathcal{N}_{G}(H) .
$$

A group $G$ is called a QTI-group if all of its subgroups are QTI-subgroups; correspondingly, $G$ is an AQTI-group if all its abelian subgroups are QTI-subgroups. It can be shown that any TI-subgroup is a QTI-subgroup, but the converse is not true. In [8], G. Qian and F. Tang classify AQTI-groups and prove that if $G$ is a $p$-group, then the properties of being TI, ATI and AQTI are equivalent in $G$.

Groups all of whose cyclic subgroups are TI-subgroups are called CTI-groups. Clearly, any ATI-group is a CTI-group; however, the converse is not true. In particular, the center of any non-nilpotent ATI-group is trivial, but this does not hold

The first author was supported by the University of Tabriz Research Affairs Office (S/27/2257-2). The third author was supported by RFBR (10-01-00324), Department of Mathematical Sciences Program of RAS (project 09-T-1-1004), joint research programs, Ural RAS to RAS (project 12- $\tilde{\mathrm{N}}-1-1018$ ) and UB RAS NAS (draft 12- $-\tilde{\mathrm{N}}-1-1009)$. 
for CTI-groups. In this paper, we classify the CTI-groups with non-trivial center. Also we prove that these groups are necessarily solvable with elementary abelian center. Next, we determine the structure of solvable CTI-groups with trivial center, and show that the centralizers of their minimal normal subgroups are equal to the Fitting subgroup of the group. Also we prove that a CTI-group is solvable if and only if it has a solvable minimal normal subgroup. Finally we classify non-solvable CTI-groups.

Our notation is standard and can be found in [2] and [11]. Throughout this paper, $F(G)$ is the Fitting subgroup of $G, Z(G)$ is the center of $G$; also $Q_{8}$ and $S_{4}$ are the quaternion group of order 8 , and the symmetric group of degree 4 , respectively.

The following easy lemmas will be useful.

Lemma 1.1. Let $G$ be a CTI-group and $H$ be a subgroup of $G$. Then:

(i) $H$ is a CTI-group.

(ii) If $H$ is cyclic and $\operatorname{Core}_{G}(H) \neq 1$, then $H \unlhd G$.

Lemma 1.2. Let $G$ be a CTI-group and assume that $x, y \in G$ have coprime orders. If $[x, y]=1$ and $\langle x\rangle \unlhd G$, then $\langle y\rangle \unlhd G$.

Proof. As $\langle x\rangle \leq\langle x y\rangle$, we have

$$
\operatorname{Core}_{G}(\langle x y\rangle) \neq 1
$$

and so $\langle x y\rangle \unlhd G$. Now since $\langle y\rangle$ is a characteristic subgroup of $\langle x y\rangle$, we have $\langle y\rangle \unlhd G$.

As an immediate corollary, we get:

Corollary 1.3. Let $G$ be a CTI-group with non-trivial center.

(i) Assume that the order of $1 \neq g \in G$ is coprime to the order of an element of $Z(G)$. Then $\langle g\rangle \unlhd G$.

(ii) If two distinct primes $p$ and $q$ divide the order of $Z(G)$, then $G$ is a Hamiltonian group.

Proof. (i) This is trivial.

(ii) Let $x \in G$ be of prime order $r$. Then, we have $(r, p)=1$ or $(r, q)=1$. Therefore by (i), $\langle x\rangle \unlhd G$, consequently any cyclic subgroup of $G$ and so any subgroup of $G$ is normal in $G$ (by Lemma 1.1 (ii)).

The preceding corollary implies that a finite non-Hamiltonian nilpotent CTIgroup is necessarily a non-abelian $p$-group. 


\section{CTI-groups with non-trivial center}

In this section, we suppose that $G$ is a non-nilpotent CTI-group with non-trivial center.

Theorem 2.1. Let $G$ be a non-nilpotent CTI-group with non-trivial center. Then $Z(G)$ is an elementary abelian p-subgroup, where $p$ is the smallest prime divisor of $|G|$. In particular, any $p^{\prime}$-subgroup of $G$ is normal.

Proof. Since $G$ is not a Hamiltonian group, it follows that $Z(G)$ is a $p$-subgroup (by Corollary 1.3 (ii)). Also Corollary 1.3 (i) implies that any $p^{\prime}$-subgroup of $G$ is normal. Now it suffices to prove that every element of $Z(G)$ is of order $p$. Let $x \in Z(G)$ satisfy $x^{p^{i}}=1$, where $i>1$. Also assume that $\langle y\rangle \not G$ is of order $p$. As $\left\langle x^{p}\right\rangle \leq\langle y x\rangle$, we have $\langle y x\rangle \unlhd G$. Therefore $\langle y x\rangle$ acts trivially on any $p^{\prime}$-element $t$ of $G$, and this implies that $[t, y]=[t, x y]=1$. Now since $\langle t\rangle \leq\langle y t\rangle$, it follows that $\langle y t\rangle \unlhd G$. Thus we conclude that $\langle y\rangle \unlhd G$ which contradicts our assumption.

Now let $q$ be the smallest prime divisor of $|G|$ and $q \neq p$. Let $y \in G$ be of order $q$. Then by Lemma 1.2, $\langle y\rangle \unlhd G$. Consequently, $y \in Z(G)$. Hence we get a contradiction and the proof is complete.

Remark 2.2. The preceding theorem states that a Hall $p^{\prime}$-subgroup of any nonnilpotent CTI-group $G$ with non-trivial center is Hamiltonian and normal, so we can write $G=H P$, where $P \in 8 \mathrm{y} \ell_{p}(G)$ and $H$ is an abelian $p^{\prime}$-subgroup, because $|H|$ is odd, since $p$ is the smallest prime divisor or $|G|$. Also we immediately see that any non-normal cyclic subgroup is necessarily a $p$-subgroup.

We continue to assume that $p$ is the smallest prime divisor of $|G|$.

Proposition 2.3. Let $G$ be a non-nilpotent $\mathrm{CTI}$-group with non-trivial center. Then for every non-normal cyclic subgroup $K$ of $G, \mathscr{C}_{G}(K)$ is a p-subgroup. In particular, $\mathcal{C}_{H}(P)=1$ and accordingly $H \leq G^{\prime}$.

Proof. Let $K=\langle x\rangle$ and $y \in \mathscr{C}_{G}(x)$ be a $p^{\prime}$-element. By Theorem 2.1, we have $\langle y\rangle \unlhd G$. Lemma 1.2 implies that $\langle x\rangle \unlhd G$ which contradicts our assumption. Therefore $\mathscr{C}_{G}(x)$ is a $p$-group and so we will have $\ell_{H}(P) \leq \boldsymbol{\ell}_{H}(x)=1$. Now the fundamental theorem of coprime actions implies that $H=[H, P]$ and hence $H \leq G^{\prime}$.

Theorem 2.4. Let $G$ be a non-nilpotent CTI-group with non-trivial center and $p$ be the smallest prime divisor of $|G|$. If $G$ has no subgroups isomorphic to a dihedral group of 2-power order, then any cyclic p-subgroup of order greater than $p$ is non-normal. 
Proof. Let $\langle x\rangle \not \exists G$ be of order $p$ and let $y \in G$ satisfy $1 \neq y^{p} \in Z(G)$. If $p=2$ and $(x y)^{2}=1$, then $y^{x}=y^{-1}$ and $\langle x, y\rangle$ is a dihedral group of 2-power order, which is contradiction. Thus

$$
(x y)^{p}=y^{p}[y, x]^{\frac{p(p-1)}{2}},
$$

since $[y, x] \in Z(G)$. Therefore $(x y)^{p}$ is a central element of $G$ and so $\langle x y\rangle \unlhd G$. Consequently, for any $p^{\prime}$-element $t$, we have $[t, x]=[t, y x]=1$ or $t \in \mathscr{C}_{G}(x)$ and this is in contradiction to Proposition 2.3.

It follows from Theorem 2.4 that if a finite non-nilpotent CTI-group has no subgroups isomorphic to a dihedral group of 2-power order, then no power of any non-trivial element of its $p$-subgroups can be central.

We can now prove our main structural theorem:

Theorem 2.5. Let $G$ be a non-nilpotent CTI-group with non-trivial center and let $p$ divide $|Z(G)|$. Then $G$ possesses an abelian $p$-subgroup $K$ such that

$$
P \cong K \rtimes \mathbb{Z}_{p^{i}}
$$

and every subgroup of $K$ is normal in $G$. Also,

(i) if $p$ is odd or $P$ is an abelian subgroup, then

$$
K=Z(G) \text { and } P=Z(G) \times \mathbb{Z}_{p^{i}},
$$

also in this case $G^{\prime} \cap Z(G)=1$,

(ii) if $p=2$ and $P$ is a non-abelian subgroup, then $i=1$ and $P$ has a subgroup isomorphic to a dihedral group of 2-power order, moreover $G^{\prime} \cap Z(G) \neq 1$,

(iii) $G^{\prime} \cap Z(G) \neq 1$ if and only if $G$ possesses a subgroup isomorphic to a dihedral group of 2-power order.

Proof. Let $h \in H$ with $|h|=q \neq p$. Then $\langle h\rangle \unlhd G$ and $P$ acts on $\langle h\rangle$ by conjugation, so there exists a homomorphism $\varphi: P \longrightarrow \operatorname{Aut}(\langle h\rangle)$.

Set $K:=\operatorname{ker} \varphi$ and let $P / K=\langle x K\rangle$. Then $P=\langle x, K\rangle$. Clearly $\langle x\rangle \not \unlhd G$, otherwise the action of $x$ on $h$ would be trivial. If for some $i, x^{i} \in K$ then we get $\langle x\rangle \unlhd G$ and this is a contradiction. Thus $\langle x\rangle \cap K=1$ and $P=K \rtimes\langle x\rangle$. As every element of $K$ commutes with $h$, by applying Lemma 1.2, we conclude that every subgroup of $K$ is normal in $G$ and therefore $K$ is a Hamiltonian group. Also it is clear that $Z(G)=\Omega_{1}(K)$.

(i) Let $p$ be odd or $P$ be an abelian group. Then $G$ has no subgroup isomorphic to a dihedral groups of 2-power order. Thus Theorem 2.4 implies that any element of $K$ is of order $p$ and so $K=Z(G)$. Hence $P=Z(G) \times \mathbb{Z}_{p^{i}}$ and $G^{\prime}=H$. Thus $G^{\prime} \cap Z(G)=1$. 
(ii) First, we note that for any $y \in K$ and $1 \neq t \in\langle x\rangle$ we have $\langle y t\rangle \not G$; otherwise $[h, t]=[h, y t]=1$ and so $t \in K \cap\langle x\rangle$, which is clearly a contradiction.

Let $y \in \mathcal{C}_{K}(x)$. If $|y| \neq 2$, then $(y t)^{2}=y^{2}$, whence $t \in\langle x\rangle$ is a element of order 2 . Therefore $\langle y t\rangle \unlhd G$, a contradiction. Consequently, $Z(G)=\mathscr{C}_{K}(x)$.

Since $P$ is non-abelian, we have $Z(G) \neq K$. Therefore, on assuming that $y \in K$ is of order 4 we see that $\left[y, x^{2}\right]=1$ (since the action of $\langle x\rangle$ on $\langle y\rangle$ is at most of order 2). Now, if $|x|=l \neq 2$ then $y^{2} \in\left\langle y x^{\frac{l}{2}}\right\rangle$ and so $\left\langle y x^{\frac{l}{2}}\right\rangle \unlhd G$. This is a contradiction; consequently, $x^{2}=1$.

Now let $y \in K$ be an arbitrary element. Since $y^{x} \in\langle y\rangle$, we have $(y x)^{2} \in K$. So, if $|y x|>2$, then we get $\langle y x\rangle \unlhd G$, a contradiction. Thus we have $|y x|=2$ and $y^{x}=y^{-1}$, in other words, $x$ inverts any element of $K$. Hence $\langle y, x\rangle$ is a dihedral group of 2-power order. So, $Z(\langle y, x\rangle) \leq G^{\prime} \cap Z(G)$.

If $K$ were a non-abelian group, then $Q_{8} \leq K$, because $K$ is a Hamiltonian group. Therefore $K$ would contain two elements $y$ and $z$ of order 4 such that $|y z|=4$ and $y^{2}=z^{2}$. But in this case we would have

$$
(y z)^{-1}=(y z)^{x}=y^{x} z^{x}=y^{-1} z^{-1}=(z y)^{-1} .
$$

Thus $[z, y]=1$ and so

$$
(z y)^{2}=z^{2} y^{2}=z^{4}=1,
$$

a contradiction. Hence, $K$ must be an abelian group.

(iii) First, let $G^{\prime} \cap Z(G) \neq 1$. Then $P$ is non-abelian. Therefore $K \neq Z(G)$, and so by (ii), $G$ has a subgroup isomorphic to $D_{2^{l}}$ for some $l$.

Conversely, assume that $P$ has a subgroup isomorphic to $D_{2^{l}}$. In this case, by (ii), $K$ has an element $y$ of order $2^{l-1}$, so $y^{2^{l-2}} \in Z(G)$ and also $y^{2^{l-2}} \in D_{2^{l}}^{\prime}$. Hence, $G^{\prime} \cap Z(G) \neq 1$.

Corollary 2.6. Let $G$ be a non-nilpotent CTI-group such that $Z(G) \neq 1$. Also suppose that $p$ divides $|Z(G)|$ and let $H$ be a Hall $p^{\prime}$-subgroup of $G$. Then $H$ is abelian and normal, and moreover $G=H P$ is solvable. Also,

(i) if $Z(G) \cap G^{\prime}=1$, then $G \cong K \times\left(H \rtimes \mathbb{Z}_{p^{i}}\right)$, where $p$ is the smallest divisor of $|G|, K=Z(G), P=Z(G) \times \mathbb{Z}_{p^{i}}$ and $H=G^{\prime}$,

(ii) if $Z(G) \cap G^{\prime} \neq 1$, then $p=2$ and $P=K \rtimes \mathbb{Z}_{2}$, where $K$ is an abelian normal subgroup of $G$; also $Z(G)=\Omega_{1}(K), G^{\prime}=H \mho^{1}(K)$ and $\mathbb{Z}_{2}$ inverts any element of $H K$,

(iii) the Fitting subgroup $F(G)=H K$ is abelian.

Lemma 2.7. Let $G$ be a non-nilpotent CTI-group with non-trivial center and let $\langle x\rangle \not \nexists G$. Then for any $y \in Z(G),\langle x, y\rangle \not \nexists G$. So the center of any non-nilpotent ATI-group is trivial. 
Proof. Assume that $\langle x, y\rangle \unlhd G$. Since any $p^{\prime}$-subgroup is normal, it follows that $x$ is a $p$-element. Therefore $\langle x, y\rangle \unlhd G$ is a $p$-subgroup of $G$, and so $x$ acts trivially on any $p^{\prime}$-element of $G$. Now, by Lemma $1.2,\langle x\rangle \unlhd G$.

Since in every ATI-group, for any $y \in Z(G)$ and $g \in G$ we have $\langle y, g\rangle \unlhd G$, and any ATI-group is a CTI-group, we get $\langle g\rangle \unlhd G$ for every $g \in G$. Hence, $G$ is Hamiltonian; a contradiction.

\section{Solvable CTI-groups with trivial center}

In this section, we show that a CTI-group $G$ is solvable if and only if it has a solvable minimal normal subgroup. Also assuming that $G$ is a solvable group with trivial center we show that if $V$ is a minimal normal subgroup of $G$, then $G \cong \mathcal{C}_{G}(V) \rtimes H$, where the Sylow subgroups of $H$ are cyclic or isomorphic to $Q_{8}$ and $F(G)=\ell_{G}(V)$. Also either $G \cong S_{4}$ or $G$ is a Frobenius group with kernel $F(G)$ and complement $H$.

We remark that if a CTI-group $G$ has a solvable minimal normal subgroup, then, by Corollary 2.6, every minimal normal subgroup of $G$ is also solvable.

Suppose that $V$ is a solvable minimal normal subgroup of $G$. As $V$ is an elementary abelian $p$-subgroup, we have $V \leq F(G)$ and so $V \leq Z(F(G))$. Hence, $F(G) \leq \ell_{G}(V)$.

Let $x \in \mathscr{C}_{G}(V)$. Then we have $V \leq \mathscr{C}_{G}(x)$. Now if $\mathscr{C}_{G}(x)$ is Hamiltonian, then $V \leq Z\left(\mathcal{C}_{G}(x)\right)$ and so $\mathscr{C}_{G}(x) \leq \mathcal{C}_{G}(V)$. If $\mathscr{C}_{G}(x)$ is non-nilpotent and $x$ is a p-element, then again $V \leq Z\left(\mathcal{C}_{G}(x)\right)$ (by Corollary 2.6), and so $\mathscr{C}_{G}(x) \leq \mathcal{C}_{G}(V)$. In particular, as $\mathcal{C}_{G}(V) \leq \mathscr{C}_{G}(x)$ for any $x \in V$, we see that if $\mathcal{C}_{G}(x)$ is Hamiltonian or non-nilpotent, then $\mathscr{C}_{G}(x)=\mathcal{C}_{G}(V)$.

For the sake of simplicity in the following theorems we set $C_{V}=\mathscr{C}_{G}(V)$, $F=F(G)$ and $C_{x}=\mathcal{C}_{G}(x)$, for any $x \in G$.

Theorem 3.1. Let $G$ be a finite CTI-group with trivial center and $V$ be a minimal normal subgroup of $G$. If $V$ is solvable, then $F=C_{V}$.

Proof. By the above discussion, it suffices to show that $C_{V}$ is nilpotent. Suppose by way of contradiction that $C_{V}$ is not nilpotent. Since $Z\left(C_{V}\right) \neq 1$, we conclude that $C_{V} \cong F \rtimes \mathbb{Z}_{p^{i}}$ where $F$ is abelian. We claim that $C_{x} \leq C_{V}$ for any $x \in C_{V}$. Therefore $G$ will be a Frobenius group with kernel $C_{V}$, and this is a contradiction, because $C_{V}$ is not nilpotent.

Consider first the case $x \in Z\left(C_{V}\right)$. Then $C_{V} \leq C_{x}$. Therefore, $C_{x}$ is also nonnilpotent and so $V \leq Z\left(C_{x}\right)$. Thus, $C_{V}=C_{x}$. Now assume that $x \notin Z\left(C_{V}\right)$. In this case, if $\langle x\rangle \unlhd C_{V}$, then $x \in F\left(C_{V}\right)=F$ and so $F \leq C_{x}$. Also either $x$ is a $p^{\prime}$-element or $p=2$ and $|x|=2^{l} \neq 2$, so in either case, $C_{x}$ is nilpotent by The- 
orem 2.1 and since it is not a $p$-group, it is a Hamiltonian group and $V \leq Z\left(C_{x}\right)$. Hence $F=C_{x} \leq C_{V}$.

Let $\langle x\rangle \not C_{V}$. If $|x|>p$, then $C_{x}$ is necessarily nilpotent. Therefore by choosing $y \in V \cap Z\left(C_{x}\right) \neq 1, C_{y}$ will be non-nilpotent because $C_{V} \leq C_{y}$. Thus we get $C_{x} \leq C_{y}=C_{V}$. Now if $|x|=p$, then either $C_{x}$ is nilpotent and so we have $V \cap Z\left(C_{x}\right) \neq 1$, or $C_{x}$ is non-nilpotent and hence $V \leq Z\left(C_{x}\right)$. So in either case, $C_{x} \leq C_{V}$. Thus $C_{V}$ is nilpotent and so $F=C_{V}$.

Notice that the Fitting subgroup of a CTI-group is not necessarily abelian. For example, using the Small Group library of GAP, we see that the group SmallGroup $(9477,4035)$, is a CTI-group with abelian center and non-abelian Fitting subgroup. The structure of this group is as follows:

$$
G \cong\left(\left(\mathbb{Z}_{3} \times \mathbb{Z}_{3} \times\left(\left(\mathbb{Z}_{3} \times \mathbb{Z}_{3}\right) \rtimes \mathbb{Z}_{3}\right)\right) \rtimes \mathbb{Z}_{3}\right) \rtimes \mathbb{Z}_{13},
$$

and its Fitting subgroup is $F(G) \cong \mathbb{Z}_{3} \times \mathbb{Z}_{3} \times\left(\left(\mathbb{Z}_{3} \times \mathbb{Z}_{3}\right) \rtimes \mathbb{Z}_{3}\right) \rtimes \mathbb{Z}_{3}$.

If the order of $F(G)$ is divisible by more than one prime, then $F(G)$ is abelian.

Proposition 3.2. Let $G$ be a finite CTI-group with trivial center and also let its minimal normal subgroup be solvable. If $|F|$ has more than one prime divisor, then $G=F H$ is a Frobenius group with abelian kernel $F$ and complement $H$.

Proof. By Corollary 1.3 (ii), $F$ is a Hamiltonian group. Therefore $F^{\prime} \leq Z(G)=1$ and so $F$ is an abelian group.

Assume that $q$ is a prime divisor of $|F|$ and $Q \in 8 \mathrm{y} \ell_{q}(G)$. As $F \cap Q \unlhd Q$, we have $F \cap Z(Q) \neq 1$. Consequently, on assuming $x \in F \cap Z(Q), C_{x}$ contains both $F$ and $Q$. Next, we show that $F$ is a Hall subgroup of $G$. First we assume that $C_{x}$ is nilpotent. Since $Q \unlhd C_{x}, Q$ commutes with a minimal normal subgroup $V$ of order coprime to $q$. Thus, $Q \leq C_{V}=F$.

Now, let $C_{x}$ be non-nilpotent. By Lemma 2.1, $C_{x}$ contains a minimal normal subgroup $V$ of $q$-power order. Also, since $V$ is elementary abelian, it follows that $V \leq Z\left(C_{x}\right)$, therefore $Q \leq C_{x} \leq C_{V}=F$. Thus, $F$ is a Hall subgroup of $G$. Consequently, $G=F H$.

Finally, to complete the proof it will suffice to show that for every $x \in F$, $C_{x} \leq F$. Let $q$ be a prime divisor of $\left|C_{x}\right|$ such that $q \nmid|F|$. Also let $y \in C_{x}$ be of order $q$. If $C_{x}$ is nilpotent, then $y \in C_{G}(F)=F$ and this is a contradiction. Now, let $C_{x}$ be non-nilpotent. Then since $x$ and $y$ have coprime orders, Corollary 2.6 (iii) implies that $y \in F\left(C_{x}\right)$ and $F\left(C_{x}\right)$ is abelian. So again $y \in C_{G}(F)=F$, because $F \leqslant F\left(C_{x}\right)$, which gives the final contradiction. Hence, $C_{x}=F$ completing the proof.

In the following theorems, we suppose that $F$ is a $p$-group. 
Lemma 3.3. Let $G$ be a CTI-group with trivial center and $K \leq G$. Also assume that a minimal normal subgroup of $G$ is solvable and $F$ is a p-group. Then:

(i) for any $x \in F, C_{x}$ is a p-group,

(ii) if $P \in 8 \mathrm{y} \ell_{p}(G)$ is maximal in $K$ and $P \not \nexists G$, then $K$ is a non-nilpotent group with trivial center. Also, $F(K)$ is a p-subgroup of $K$ and $P \not K$.

Proof. (i) Let $V$ be a minimal normal subgroup of $G$ and $x \in F$. Suppose that $C_{x}$ is not a $p$-group. Since any $p^{\prime}$-subgroup of $C_{x}$ is normal, whether $C_{x}$ is or is not nilpotent, we see that $F=C_{V}$ contains a $p^{\prime}$-element (because $V \leqslant C_{x}$ ) and this is a contradiction. Hence for any $x \in F(G)$, we observe that $C_{x}$ is a $p$-group.

(ii) Suppose $K \leq G$ contains $P$ as a maximal subgroup. Then $V \leq F \leq F(K)$. Now since for every $x \in F$, the subgroup $C_{x}$ is a $p$-group, so is $F(K)$. Therefore, $F(K)=\operatorname{Core}_{G}(P)=F$. Thus $K$ is non-nilpotent and also $Z(K)=1$ (otherwise, since $Z(K) \leq F$, for any $x \in Z(K), K \leq C_{x}$ would be a $p$-group).

Theorem 3.4. Let $G$ be a finite solvable CTI-group with trivial center. Assume further that $F$ is a p-group. Then either $G$ is isomorphic to $S_{4}$, or $F$ is a Sylow p-subgroup of $G$ and $G$ is a Frobenius group with kernel $F$.

Proof. Let $P$ be a Sylow $p$-subgroup of $G$. If $P$ is normal in $G$, then $F=P$ is the Frobenius kernel and the desired conclusion follows. So let $P \not \nexists G$. We shall show $G \cong S_{4}$.

Assume now that $P$ is a maximal subgroup of $K \leq G$. By the preceding lemma, we have $Z(K)=1$ and $P \not K$. Now, if the conclusion is established for $K$ namely, $K \cong S_{4}$, then $F \cong \mathbb{Z}_{2} \times \mathbb{Z}_{2}$. Thus, we get $S_{3} \cong K / F \leq G / F \hookrightarrow S_{3}$, therefore $K=G$. Hence without loss of generality we may assume that $P$ is maximal in $G$.

Let $Q$ be a Sylow $q$-subgroup of $G$, whence $q \neq p$. Then $Q F$ is a Frobenius group with kernel $F$. Therefore $Q$ is either cyclic or generalized quaternion. As $P$ is a maximal subgroup of $G$, we have $G=P Q$, furthermore, $Q F / F$ is a unique minimal normal subgroup of $G / F$, because $F=\operatorname{Core}_{G}(P)$. Hence we will have $Q \cong \mathbb{Z}_{q}$ and so $q \neq 2$ (otherwise, $P \unlhd G$ ). Also, $P / F \hookrightarrow \operatorname{Aut}(Q)$. Thus $P / F$ is cyclic and $p \mid q-1$.

Now, set $N=\mathcal{N}_{G}(Q)$. Then by the Frattini argument, we have $G=N F$, because $Q F \unlhd G$. If $F \cap N \neq 1$, then since $[F \cap N, Q]=1$, we will have $Q \leq C_{x}$, for any $x \in N \cap F$ and this is a contradiction, since $C_{x}$ is a $p$-group. Thus, we obtain $F \cap N=1$ and so $Q \supsetneqq N$. Let $P_{1}$ be a Sylow $p$-subgroup of $N$. Then $P_{1}$ is cyclic and $N=Q P_{1}$ is a CTI-group. As $F Z(N) \unlhd G$, we have

$$
Z(N) \leq F \cap N=1
$$

so $\operatorname{Core}_{N}\left(P_{1}\right)=1$, therefore $\left|P_{1}\right| \mid q-1$. 
Assume that $V$ is a minimal normal subgroup of $G$ and also $a$ and $x$ are generators of $P_{1}$ and $Q$, respectively.

Step 1. $e_{F}(a) \cap\left(C_{F}(a)\right)^{x}=1$ and so $Z(P) \cap Z\left(P^{x}\right)=1$.

Assume that $f \in \mathcal{C}_{F}(a) \cap\left(\boldsymbol{C}_{F}(a)\right)^{x}$. Then there exists an element $f_{1} \in \mathcal{C}_{F}(a)$ such that $f=f_{1}^{x}$. Therefore $f_{1}^{x}=\left(f_{1}^{x}\right)^{a}=f_{1}^{x^{a}}$ and so $f_{1} \in \mathcal{C}_{F}([x, a])=1$, because $[x, a] \in Q$.

Step 2. $p=2$ and $\left|\left(V P_{1}\right)^{\prime}\right|=\left|P_{1}\right|=2$.

Let $\left|P_{1}\right|=p^{m}$ and $z \in Z(P) \cap V$ be of order $p$. We set $z_{i}=z^{x^{i}}$, for any $i \geq 0$. Then $C=\left\{z_{i} \mid 0 \leq i<q\right\}$ is the set of conjugates of $z$ by $Q$. The set $C$ is also invariant under conjugation by $P_{1}$ and if for some $l \neq 0$ and $i>1, z_{i}^{a^{l}}=z_{i}$, then $z^{x^{i}}=z^{a^{-l} x^{i} a^{l}}$. Thus

$$
a^{-l} x^{i} a^{l} x^{-i} \in e_{Q}(z)=1,
$$

so $a^{-l} x^{i} a^{l}=x^{i}$ then $a^{l} \in \operatorname{Core}_{N}\left(P_{1}\right)=1$, which is a contradiction. Consequently, only the element $z=z_{0}$ of $C$ is invariant under the action of $P_{1}$. Therefore, we have

$$
C=\{z\} \cup \bigcup_{l=1}^{k} \operatorname{Orbit}_{P_{1}}\left(z_{i_{l}}\right) .
$$

Now, let $u=\prod_{i=0}^{q-1} z_{i}$. Since $u^{x}=u$, we have $u \in \mathscr{C}_{F}(x)=1$. Thus

$$
1=\prod_{i=0}^{q-1} z_{i}=z \prod_{l=1}^{k} \prod_{t \in \operatorname{Orbit}_{P_{1}}\left(z_{i_{l}}\right)} t .
$$

If $\exp \left(V P_{1}\right)=p^{m}$, then

$$
1=\left(a^{-1} z_{i}\right)^{p^{m}}=\prod_{l=1}^{p^{m}} z_{i}^{a^{l}}=\prod_{t \in \operatorname{Orbit}_{P_{1}}\left(z_{i}\right)} t .
$$

By (*), $z=1$ and this is a contradiction. Thus there exists a $z_{i} \in C$ such that $a^{-1} z_{i}$ is of order $p^{m+1}$. Since $a^{-1} z_{i} \notin V$, it follows that $v=\left(a^{-1} z_{i}\right)^{p^{m}}$ belongs to the center of $V P_{1}$, therefore $\left\langle a^{-1} z_{i}\right\rangle \unlhd V P_{1}$ (VP 1 is a CTI-group). Also we will have

$$
V P_{1} /\langle v\rangle \cong V /\langle v\rangle \times\left\langle a^{-1} z_{i}\right\rangle /\langle v\rangle .
$$

Thus $\left[V P_{1}, V P_{1}\right]=\langle v\rangle \leq Z\left(V P_{1}\right)$ and so

$$
\left(a z_{i}\right)^{p}=a^{p} z_{i}^{p}\left[z_{i}, a\right]^{p(p-1) / 2} .
$$

If $p$ is odd or $m>1$, then we have $\left(a z_{i}\right)^{p^{m}}=a^{p^{m}}=1$ and this a contradiction. Hence, $p=2, m=1$ and $\left|P_{1}\right|=2$. 
Step 3. $V \cong \mathbb{Z}_{2} \times \mathbb{Z}_{2}, q=3$ and $V N \cong S_{4}$.

We set $Z=Z\left(V P_{1}\right)$. Then $Z \cap Z^{x}=1$ by step 1 . Since $C \subseteq Z(F(G))$, we have $\langle C\rangle \unlhd G$ therefore $V=\langle C\rangle$. Since for any $i>1,\left[z_{1}, a\right]=\left[z_{i}, a\right]$, it follows that $z_{1} z_{i}^{-1} \in Z$; consequently, $V / Z \cong\left\langle z_{1}\right\rangle$ and so $Z^{x} \cong \mathbb{Z}_{2}$ and $V \cong \mathbb{Z}_{2} \times \mathbb{Z}_{2}$. Hence $q=3$ and $V N \cong S_{4}$.

Step 4. $F(G)$ is the unique minimal normal subgroup of $G$ and thus $G \cong S_{4}$.

Let $z_{1}$ and $z_{2}$ be two distinct central elements of order 2. Then for $v_{1}=z_{1}^{x}$ and $v_{2}=z_{2}^{x}$, the subgroups $V_{1}=\left\langle z_{1}, v_{1}\right\rangle$ and $V_{2}=\left\langle z_{2}, v_{2}\right\rangle$ will be two distinct minimal normal subgroup of $G$. Thus $v_{1}^{a}=z_{1} v_{1}$ and $v_{2}^{a}=z_{2} v_{2}$, and also

$$
\left(a v_{1}\right)^{v_{2}}=v_{2} a v_{1} v_{2}=a v_{1} z_{2} \text {. }
$$

Since $P$ is a CTI-group and $\left(a v_{1}\right)^{2}=\left(a v_{1} z_{2}\right)^{2}=z_{1}$, we will have

$$
a v_{1} z_{2}=\left(a v_{1}\right)^{3}=a v_{1} z_{1}
$$

and so $z_{1}=z_{2}$, a contradiction. Thus $Z(P)$ is cyclic and therefore $G$ possesses a unique minimal normal subgroup $\langle z, v\rangle$, where $z \in Z(P)$ and $v \in V$.

As $(v a)^{2}=z$, we have $\langle v a\rangle \unlhd P$ and so $[F,\langle v a\rangle] \leq F \cap\langle v a\rangle=\langle z\rangle$. Since for every $f \in F,[f, v]=1$, we will have $[F, a] \leq\langle z\rangle$ and so $F^{2} \leq \mathscr{C}_{F}(a)$; consequently, $\mathscr{C}_{F}(a) \unlhd F$ and $F / \mathscr{C}_{F}(a)$ is elementary abelian.

Finally assume that $f_{1}, f_{2} \notin \mathscr{C}_{F}(a)$. Then we have $f_{2}^{-1} f_{1} \in \mathscr{C}_{F}(a)$, because $\left[f_{1}, a\right]=\left[f_{2}, a\right]$. Therefore, $F / \complement_{F}(a)$ is cyclic and so it is isomorphic to $\mathbb{Z}_{2}$. By step 1 , we have $\left|\mathcal{C}_{F}(a)\right|=\left|\boldsymbol{C}_{F}(a)^{x}\right|=2$, consequently, $F=V \cong \mathbb{Z}_{2} \times \mathbb{Z}_{2}$ and the desired conclusion follows.

Theorem 3.5. Let $G=K H$ be a finite Frobenius CTI-group with kernel $K$ and complement $H$. Then,

(i) if $|H|$ is odd, then $H$ is cyclic,

(ii) if $|H|$ is even, then $K$ is abelian and either $H$ is cyclic or $H \cong Q_{8} \times \mathbb{Z}_{n}$, where $n$ is odd.

In either case $G$ is solvable.

Proof. (i) Since $H$ is a solvable group and cannot be Frobenius group by [10, Theorem 12.6.11], it follows that $Z(H) \neq 1$ by Theorem 3.4 and 3.2. Now by Corollary 2.6, $H$ is a nilpotent. Therefore $H$ is cyclic by [2, Theorem 10.3.1 (iv)].

(ii) By [2, Theorem 10.3.1 (iii), (iv)], $K$ is abelian and $Z(H) \neq 1$ again by Corollary 2.6, $H$ is nilpotent. We can easily see that the only generalized quaternion CTI-group is $Q_{8}$. Therefore either $H$ is a cyclic group or $H \cong Q_{8} \times \mathbb{Z}_{n}$, where $n$ is odd.

Theorem 3.6. A CTI-group $G$ is solvable if and only if it has a solvable minimal normal subgroup. 
Proof. If $Z(G) \neq 1$ or $F(G)$ is not a $p$-group, then by Proposition 3.2 and Corollary 2.6, $G$ is solvable. So we assume that $Z(G)=1$ and $F(G)$ is a $p$-group.

Let $G$ be a minimal counterexample for the theorem. Let $P \in 8 \mathrm{y} \ell_{p}(G)$. By Theorems 3.5 and 3.4, $P \Varangle G$. Suppose that a proper subgroup $K$ of $G$ contains $P$ as a maximal subgroup. Therefore we have $P \Varangle K, F(K)=F(G)$ and $Z(K)=1$ (by Lemma 3.3), also by the choice of $G, K$ is solvable and so $K \cong S_{4}$. Hence $P \cong D_{8}$ and $F(G) \cong \mathbb{Z}_{2} \times \mathbb{Z}_{2}$. Therefore $G / F(G)$ is solvable which is a contradiction. And so $P$ is a maximal subgroup of $G$. By a well-known theorem of Thompson [2, Theorem 10.3.2], $p=2$ and by [9, Theorem II], $G / F$ has a unique minimal normal subgroup $K / F$ such that $G / K$ is a 2-group. Hence $K$ is not solvable. Again by the minimality of $G$, we have $K=G$. Now by [5, Theorem 2.13] every involution of $G / F$ inverts an element of odd order in $G / F$, so $G / F$ contains a non-nilpotent dihedral subgroup. Consider the inverse image $R$ of this dihedral subgroup in $G$. Obviously $Z(R)=1$ and $R$ is solvable with non-normal Sylow 2-subgroup. By using Theorem 3.4, $R \cong S_{4}$ and $F$ is a four group and this is also a contradiction.

\section{Non-solvable CTI-groups}

In this section we classify non-solvable CTI-groups. Let $V$ be a minimal normal subgroup of a non-solvable CTI-group $G$. By Theorem 3.6, $V$ cannot be solvable, since the centralizer of any element (in particular any subgroup) of $G$ is solvable, and so $\ell_{G}(V)=1$. Therefore, $V$ must be simple. Also we have

$$
V \leq G \hookrightarrow \operatorname{Aut}(V) \text { and } \quad G / V \hookrightarrow \operatorname{Out}(V) \text {. }
$$

Lemma 4.1. Let $G$ be a non-solvable CTI-group with minimal normal subgroup $V$ and $P \in \delta \mathrm{y} \ell_{2}(V)$. If $N=\mathcal{N}_{G}(P)$ is non-nilpotent, then $Z(N)=1$.

Proof. If $Z(N) \neq 1$, then by Corollary 2.6 either $P \leqslant Z(N)$ or $\ell_{G}(P)$ has index 2 in $N$. In the latter case, we have $\mathcal{N}_{V}(P)=\mathcal{C}_{V}(P)$. In either case, we get $P \leqslant Z\left(\mathcal{N}_{V}(P)\right)$ and so $P$ has a normal $p$-complement in $V$, a contradiction.

Theorem 4.2. Let $G$ be a finite non-solvable CTI-group. Then $G \cong \operatorname{PSL}(2, q)$ or $G \cong \operatorname{PGL}(2, q)$, where $q>3$ is a prime power.

Proof. Let $G$ be a finite non-abelian simple CTI-group. Since every $p$-local subgroup of $G$ is solvable, then $G$ is an N-group. Now by a theorem of Thompson ([2, Theorem, p. 474]), only the groups $\operatorname{PSL}(2, q)$ and $\operatorname{Sz}(q)$ which do not contain $\operatorname{SL}(2,3)$ can be CTI (because $\operatorname{SL}(2,3)$ is not a CTI-group). Let $G \cong \operatorname{Sz}(q)$ and $P \in 8 \mathrm{y} \ell_{2}(G)$. Then by [1, Lemma 1 and Proposition 3] we have $\Omega_{1}(P)=Z(P)$ 
and $|P|=|Z(P)|^{2}$. Since $P$ is a non-abelian CTI-group, $P$ must be a non-abelian Hamiltonian group of order 16 . This is a contradiction.

Now we consider the non-simple case: then $G$ is isomorphic to a subgroup of $H=\operatorname{Aut}(\operatorname{PSL}(2, q))=\operatorname{PGL}(2, q) \rtimes\langle x\rangle$, where $q=p^{f}$ and $x$ has order $f$. Let $g \in G \backslash \operatorname{PGL}(2, q)$ be a power of $x$. Then $f \neq 1$ also $\operatorname{PSL}(2, p) \leqslant \mathcal{C}_{G}(g)$, because $\mathcal{C}_{H}(x)=\operatorname{PGL}(2, p) \times\langle x\rangle$. Since $\mathcal{C}_{G}(g)$ is non-Hamiltonian and solvable, it follows that $|g|=2$ (by Corollary 2.6), and $p=2$, because a Sylow 3-subgroup of PSL $(2,3)$ is non-normal. Now let $S \in 8 \mathrm{y} \ell_{2}(G)$ and $P \in 8 \mathrm{y} \ell_{2}(\operatorname{PGL}(2, q))$ such that $P \leqslant S$. Then $S=P\langle g\rangle$. Suppose $N=\mathcal{N}_{G}(P)$; by Lemma 4.1, $Z(N)=1$. If $S \unlhd N$, then $N=S\langle y\rangle$, where $|y|=q-1$ (by [2, Lemma 15.1.1]). Hence $[g, y]=1$ and $N$ cannot be a Frobenius group; now by Theorem 3.4, $N \cong S_{4}$ and $f=2$. Therefore, $G \cong \operatorname{Aut}(\operatorname{PSL}(2,4))$ which is isomorphic to $\operatorname{PGL}(2,5)$.

In the other case, since $G$ is a pre-image of a subgroup of

$$
\operatorname{Out}(\operatorname{PSL}(2, q))=\langle\bar{y}\rangle \times\langle\bar{x}\rangle, \quad \text { where }|y|=(2, q-1),
$$

then either $G$ is isomorphic to $\operatorname{PGL}(2, q)$, where $q>3$ is a prime power or $p$ is odd, $f$ is even and $G \cong\left\langle\operatorname{PSL}(2, q), y x^{f / 2}\right\rangle$. In the latter case $G$ is isomorphic to a non-solvable maximal subgroup of $\operatorname{PGL}(2, q) \rtimes\left\langle x^{f / 2}\right\rangle$. Now by [3, Lemma 6.6.3], $G$ is isomorphic to PGL ${ }^{*}(2, q)$ which has semidihedral Sylow 2-subgroup. This case cannot occur because a semidihedral group is not CTI.

The inverses of Corollary 2.6 and Theorem 3.4 are simple: we just prove the inverse of the non-solvable case. Before proving the inverse theorem, we consider the simple fact that if a non-normal subgroup $\langle x\rangle$ of $G$ is normal in a non-normal maximal subgroup $M$, then $\langle x\rangle \cap\langle x\rangle^{g} \unlhd G$, where $g \in G \backslash M$.

Theorem 4.3. Let $G$ be isomorphic to $K$, where $\operatorname{PSL}(2, q) \leq K \leq \operatorname{PGL}(2, q)$, $q>3$ is a power of prime $p$. Then $G$ is a CTI-group.

Proof. We can simply check by GAP that $\operatorname{PSL}(2, p)$ is CTI for $p=5,7,9,11$. Let $x$ be an element of $G$. If $p|| x \mid$, then $x$ must be a $p$-element, because by [2, Lemma 15.1.1] Sylow $p$-subgroups of $G$ are elementary abelian and TI; therefore $|x|=p$. If $|x| \mid\left(q^{2}-1\right)$ and $x$ is not a 2-element, then $|x| \mid 2^{n} m$, where $m$ is odd; hence $x=y z$, where $|z|>1$ is odd. In this case $z$ belongs to the maximal subgroup $D_{2(q-1)}$ or $D_{2(q+1)}$ by [7, Theorem 2.1 and Theorem 2.2]; since $\langle z\rangle$ is normal in these groups, it follows that $\mathcal{N}_{G}(x)=\mathcal{N}_{G}(z)$ is a non-normal maximal subgroup of $G$. Therefore, $\langle x\rangle$ is normal in a non-normal maximal subgroup of $G$, and so is TI. Now, let $x$ be a 2-element and $|x|>2$; then $p$ is an odd prime and again $\langle x\rangle$ belongs to the dihedral group. Since $\langle x\rangle$ is normal in this group, it follows that $\mathcal{N}_{G}(x)$ is maximal in $G$. Hence $\langle x\rangle$ is a TI-group. Therefore, $G$ is a CTI-group. 


\section{Bibliography}

[1] M. J. Collins, The characterisation of the Suzuki groups by their Sylow 2-subgroups, Math. Z. 123 (1971), 32-48.

[2] D. Gorenstein, Finite Groups, Chelsea, New York, 1980.

[3] D. Gorenstein and K. Harada, Finite groups whose 2-subgroups are generated by at most 4 elements, Mem. Amer. Math. Soc. 174 (1974).

[4] X. Guo, S. Li and P. Flavell, Finite groups whose abelian subgroups are TI-subgroups, J. Algebra 307 (2007), 565-569.

[5] I. M. Isaacs, Finite Group Theory, American Mathematical Society, Providence, 2008.

[6] S. Li and X. Guo, Finite $p$-groups whose abelian subgroups have a trivial intersection, Acta Math. Sin. (Engl. Ser.) 23 (2007), 731-734.

[7] A. Lucchini and A. Maróti, On finite simple groups and Kneser graphs, J. Algebraic Combin. 30 (2009), 549-566.

[8] G. Qian and F. Tang, Finite groups all of whose Abelian subgroups are QTI-subgroups, J. Algebra 320 (2008), 3605-3611.

[9] J. S. Rose, On finite insoluble groups with nilpotent maximal subgroups, J. Algebra 48 (1977), 182-196.

[10] W. R. Scott, Group Theory, Prentice-Hall, Englewood Cliffs, 1964.

[11] M. Suzuki, Group Theory II, Springer-Verlag, New York, 1986.

[12] The GAP Group, GAP-Groups. Algorithms and Programming, Version 4.4, 2005.

[13] G. Walls, Trivial intersection groups, Arch. Math. (Basel) 32 (1979), 1-4.

Received June 7, 2012; revised September 11, 2012.

\section{Author information}

Hamid Mousavi, University of Tabriz, Department of Mathematics,

P. O. Box 51666-17766, Tabriz, Iran.

E-mail: hmousavi@tabrizu.ac.ir

Tahereh Rastgoo, Institute for Advanced Studies in Basic Sciences,

Department of Mathematics, P. O. Box 1159-45195, Zanjan, Iran.

E-mail: rastgoo@iasbs.ac.ir

Viktor Zenkov, Institute of Mathematics and Mechanics of Ural Branch RAS,

S. Kovalevskaya 16, Ekaterinburg 620990, Russia;

and Ural Federal University, Energy Institute, Mira 19, Ekaterinburg 620290, Russia.

E-mail: v1i9z52@mail.ru 
Copyright of Journal of Group Theory is the property of De Gruyter and its content may not be copied or emailed to multiple sites or posted to a listserv without the copyright holder's express written permission. However, users may print, download, or email articles for individual use. 